\title{
Boolean-Valued Second-Order Logic
}

\author{
Daisuke Ikegami and Jouko Väänänen
}

\begin{abstract}
In so-called full second-order logic, the second-order variables range over all subsets and relations of the domain in question. In so-called Henkin second-order logic, every model is endowed with a set of subsets and relations which will serve as the range of the second-order variables. In our Booleanvalued second-order logic, the second-order variables range over all Booleanvalued subsets and relations on the domain. We show that under large cardinal assumptions Boolean-valued second-order logic is more robust than full secondorder logic. Its validity is absolute under forcing, and its Hanf and Löwenheim numbers are smaller than those of full second-order logic.
\end{abstract}

\section{Introduction}

Second-order logic is notoriously difficult to axiomatize. The set of Gödel numbers of valid second-order sentences, even in the empty vocabulary, is $\Pi_{2}$-complete in the Levy-hierarchy (see Väänänen [15]). One way to remedy this is the introduction of the so-called Henkin models, that is, models $\mathfrak{U}$ in which the range of second-order variables is limited to a set $\mathcal{E}$ of sets and relations, where the set $\mathcal{E}$ is allowed to vary with the model. Thus a Henkin model is a pair $(\mathfrak{U}, \mathscr{E})$, and ordinary models correspond to the case that $\mathcal{G}$ contains all sets and relations on $A$. Extending the concept of a model in this way solves the problem of axiomatizability, in that there is an obvious axiomatization which satisfies a completeness theorem with respect to models by Henkin [3].

This paper is devoted to a different modification of second-order logic. Rather than allowing the second-order variables to range over fewer than all relations, we allow them to range over more than all relations by allowing also Boolean-valued relations. This modification does not change second-order logic as much as allowing Henkin models does. In fact, in many respects the Boolean-valued second-order

Received May 9, 2012; accepted December 9, 2013

2010 Mathematics Subject Classification: Primary 03-06; Secondary 03C95, 03E40, 03E57

Keywords: Boolean-valued second-order logic, full second-order logic, $\Omega$-logic, Boolean validity

(C) 2015 by University of Notre Dame 10.1215/00294527-2835065 
logic is closer to the original second-order logic than to the Henkin second-order logic. We investigate in particular the decision problem, the completeness theorem, forcing absoluteness, the compactness theorem, the Hanf number, and the Löwenheim number.

Boolean-valued models for set theory were developed by Scott and Solovay. We refer to the monograph of Bell [2] for an exposition of the Scott-Solovay construction of a Boolean-valued universe of sets. The foreword of Bell's book by Scott contains an account of the history of the invention of Boolean-valued models of set theory. Scott refers, in particular, to the book The Mathematics of Metamathematics by Rasiowa and Sikorski [9] and asks why Boolean-valued second-order logic was not considered by the researchers whose work is reported in that book. In hindsight, given the success of Boolean-valued logic in set theory, it seems most natural to consider also Boolean-valued second-order logic.

It seems reasonable to think of second-order logic as a fragment of set theory, more or less the " $\Sigma_{2}$-fragment" of set theory (see Väänänen [15], [16]). Thus a lot of Boolean-valued set theory translates into Boolean-valued second-order logic and vice versa. The question arises: What does Boolean-valued second-order logic offer over and above Boolean-valued set theory? The point is that Boolean-valued second-order logic is a logic in the same sense that applies to first-order logic, infinitary logic, and logics with generalized quantifiers. Therefore we can use Booleanvalued second-order logic to study a multitude of structures (i.e., groups, fields, partial orders, and so forth, not just set theory). The difference between Boolean-valued second-order logic and Boolean-valued set theory is similar to the difference between second-order logic and set theory (see, e.g., [16]).

A recent development in Boolean-valued set theory is the emergence of Woodin's $\Omega$-logic (see [17]). We use $\Omega$-logic as a model for obtaining a proof concept for Boolean-valued second-order logic which would be more reasonable than the semantic consequence relation yet at the same time closer to the semantic consequence relation than the mere comprehension axioms (and the axioms of choice). Our concept of Boolean provability (in second-order logic) is an adaptation of Woodin's concept of $\Omega$-provability. Throughout we rely heavily on [17] (see also Bagaria, Castells, and Larson [1] and Larson [5] on $\Omega$-logic).

Validity in classical second-order logic is not forcing absolute. This is most blatantly revealed by the well-known fact that the continuum hypothesis can be expressed as the validity of a sentence of second-order logic. That is, there is a second-order sentence $\theta$ (in the empty language) so that $\theta$ is valid if and only if the continuum hypothesis holds. Assuming a proper class of Woodin cardinals, as in $\Omega$-logic, validity in the Boolean-valued second-order logic is forcing absolute. With the further assumption of the $\Omega$-conjecture, we obtain, as in $\Omega$-logic, the result that the set of Gödel numbers of valid sentences in Boolean-valued second-order logic is $\Delta_{2}$. Recall that the set of Gödel numbers of valid sentences in 2-valued second-order logic is $\Pi_{2}$-complete, so we have an improvement from $\Pi_{2}$ to $\Delta_{2}$.

Once we have defined our own concept of Boolean provability, an adaptation from Woodin's concept of $\Omega$-provability, we obtain the second-order version of the $\Omega$-conjecture. This version implies Woodin's $\Omega$-conjecture, but we do not know whether they are equivalent. In fact we do not even know if our version of the $\Omega$-conjecture is consistent. 
To further estimate the relationship between our Boolean-valued second-order logic and the original 2-valued second-order logic, we investigate three invariants of logics:

- The compactness number (i.e., the least $\kappa$ so that any theory in the logic, all subsets of size less than $\kappa$ of which have a model, has a model).

- The Hanf number (i.e., the least $\kappa$ so that any sentence in the logic which has a model of cardinality at least $\kappa$ has arbitrarily large models).

- The Löwenheim number (i.e., the least $\kappa$ so that any sentence in the logic which has a model, has a model of cardinality at most $\kappa$ ).

In the case of first-order logic these invariants are all $\omega$, and the same is true of the Henkin second-order logic. For the original 2-valued second-order logic these numbers are quite large (see Magidor [6]). The Hanf number is the supremum of all $\Sigma_{2}$-definable ordinals, and the Löwenheim number is the supremum of all $\Pi_{2}$-definable ordinals (see Väänänen [14]). We show that if certain large cardinals exist, then these invariants are smaller for the Boolean-valued second-order logic than what they are for the 2-valued second-order logic. In particular, we show the following.

- Assuming a proper class of Woodin cardinals, a strong form of the $\Omega$ conjecture, and the existence of supercompact cardinals, the first supercompact cardinal is above the compactness number of Boolean-valued secondorder logic and below the compactness number of 2-valued second-order logic.

- Assuming a proper class of Woodin cardinals, a strong form of the $\Omega$ conjecture, and the existence of supercompact cardinals, the first supercompact cardinal is above the Hanf number of Boolean-valued second-order logic and below the Hanf number of 2-valued second-order logic.

- Assume that the theory ZFC + "There is a proper class of Woodin cardinals." Then there is a model of set theory with a proper class of Woodin cardinals such that the first Woodin cardinal is above the Löwenheim number of Boolean-valued second-order logic and below the Löwenheim number of 2-valued second-order logic.

\section{Boolean-Valued Second-Order Logic, Semantics, and Boolean Validity}

Definition 2.1 (Boolean-valued structures) $\quad$ Let $\mathscr{L}=\left\{R_{1}, \ldots, R_{n}\right\}$ be a relational language. A Boolean-valued $\mathscr{L}$-structure consists of $M=\left(A, \mathbb{B},\left\{R_{i}^{M}\right\}_{1 \leq i \leq n}\right)$, where

(1) $A$ is a nonempty set,

(2) $B$ is a complete Boolean algebra, and

(3) for each $1 \leq i \leq n$, if $R_{i}$ is an $m$-ary relation symbol, then $R_{i}^{M}$ is a function from $A^{m}$ to $\mathbb{B}$.

When $\mathbb{B}=\{0,1\}$, a Boolean-valued $\mathscr{L}$-structure can be seen as a first-order $\mathscr{L}$-structure by identifying $R_{i}^{M}$ with the characteristic function of an $m$-ary predicate.

We now interpret second-order sentences by Boolean-valued structures in the following way. 
Definition 2.2 Let $\mathscr{L}$ be as above, and let $M=\left(A, \mathbb{B},\left\{R_{i}^{M}\right\}_{1 \leq i \leq n}\right)$ be a Boolean-valued $\mathscr{L}$-structure. For each second-order $\mathscr{L}$-formula $\varphi, \vec{a} \in A^{<\omega}$, and $\vec{f} \in\left(\mathbb{B}^{A}\right)^{<\omega}$, we assign $\|\varphi[\vec{a}, \vec{f}]\|^{M}$ by induction on the complexity of $\varphi$ in the following way:

(1) $\varphi$ is $x=y$, where $x, y$ are first-order variables; then $\|x=y[a, b]\|^{M}$ is 1 if $a=b$ and 0 otherwise.

(2) $\varphi$ is $R_{i}(x)$, where $x$ is a first-order variable; then $\left\|R_{i}(x)[a]\right\|^{M}=R_{i}^{M}(a)$.

(3) $\varphi$ is $X(x)$, where $X$ is a second-order variable and $x$ is a first-order variable; then $\|X(x)[a, f]\|^{M}=f(a)$.

(4) $\varphi$ is $\neg \psi$; then $\|\varphi[\vec{a}, \vec{f}]\|^{M}=1-\|\psi[\vec{a}, \vec{f}]\|^{M}$.

(5) $\varphi$ is $\psi_{1} \wedge \psi_{2}$; then $\|\varphi[\vec{a}, \vec{f}]\|^{M}=\left\|\psi_{1}[\vec{a}, \vec{f}]\right\|^{M} \wedge\left\|\psi_{2}[\vec{a}, \vec{f}]\right\|^{M}$.

(6) $\varphi$ is $(\exists x) \psi$, where $x$ is a first-order variable; then

$$
\|\varphi[\vec{a}, \vec{f}]\|^{M}=\bigvee_{b \in A}\|\psi[b, \vec{a}, \vec{f}]\|^{M} .
$$

(7) $\varphi$ is $(\exists X) \psi$, where $X$ is a second-order variable; then

$$
\|\varphi[\vec{a}, \vec{f}]\|^{M}=\bigvee_{g: A \rightarrow \mathbb{B}}\|\psi[\vec{a}, g, \vec{f}]\|^{M} .
$$

Note that interpreting second-order formulas via Boolean-valued structures is the same as doing it via full second-order structures in set-generic extensions.

Definition 2.3 Let $\mathscr{L}$ be as above, and let $M=\left(A, \mathbb{B},\left\{R_{i}^{M}\right\}_{1 \leq i \leq n}\right)$ be a Booleanvalued $\mathscr{L}$-structure. Let $G$ be a $\mathbb{B}$-generic filter over $V$. Then we define the full second-order $\mathscr{L}$-structure $M[G]$ in $V[G]$ as follows:

- the first-order part of $M[G]$ is $A$,

- the second-order part of $M[G]$ is $\bigcup_{n \in \omega} \mathcal{P}\left(A^{n}\right)^{V[G]}$, and

- the interpretation of $R_{i}$ is

$$
R_{i}^{M[G]}=\left\{\vec{x} \in A^{m_{i}} \mid R_{i}^{M}(\vec{x}) \in G\right\},
$$

where $m_{i}$ is the arity of $R_{i}$.

Lemma 2.4 Let $\mathscr{L}$ be as above, and let $M=\left(A, \mathbb{B},\left\{R_{i}^{M}\right\}_{1 \leq i \leq n}\right)$ be a Booleanvalued $\mathscr{L}$-structure. Let $G$ be a $\mathbb{B}$-generic filter over $V$. Then for each second-order $\mathscr{L}$-formula, $\vec{a} \in A^{<\omega}$, and $\vec{f} \in\left(\mathbb{B}^{A}\right)^{<\omega}$, we have

$$
\|\varphi[\vec{a}, \vec{f}]\|^{M} \in G \Longleftrightarrow M[G] \vDash \varphi\left[\vec{a}, \vec{f}^{G}\right],
$$

where $\vec{f}^{G}=\left\{f_{1}^{G}, \ldots, f_{n}^{G}\right\}$ and for each $i$ with $1 \leq i \leq n$,

$$
f_{i}^{G}=\left\{x \in A \mid f_{i}(x) \in G\right\} .
$$

Proof We show the statement by induction on the complexity of $\varphi$.

Case 1: $\varphi$ is $x=y$, where $x, y$ are first-order variables. Then

$$
\begin{aligned}
\|\varphi[a, b]\|^{M} \in G & \Longleftrightarrow a=b \\
& \Longleftrightarrow M[G] \vDash \varphi[a, b] .
\end{aligned}
$$


Case 2: $\varphi$ is $R_{i}(x)$, where $x$ is a first-order variable. Then

$$
\begin{aligned}
\|\varphi[a]\|^{M} \in G & \Longleftrightarrow R_{i}^{M}(a) \in G \\
& \Longleftrightarrow a \in R_{i}^{M[G]} \\
& \Longleftrightarrow M[G] \vDash \varphi[a] .
\end{aligned}
$$

Case 3: $\varphi$ is $X(x)$, where $X$ is a second-order variable and $x$ is a first-order variable. Then

$$
\begin{aligned}
\|\varphi[a, f]\|^{M} \in G & \Longleftrightarrow f(a) \in G \\
& \Longleftrightarrow M[G] \vDash \varphi\left[a, f^{G}\right] .
\end{aligned}
$$

Case 4: $\varphi$ is $\neg \psi$. Then

$$
\begin{aligned}
\|\varphi[\vec{a}, \vec{f}]\|^{M} \in G & \Longleftrightarrow\|\psi[\vec{a}, \vec{f}]\|^{M} \notin G \\
& \Longleftrightarrow M[G] \not \models \psi\left[\vec{a}, \vec{f}^{G}\right] \\
& \Longleftrightarrow M[G] \vDash \varphi\left[\vec{a}, \vec{f}^{G}\right] .
\end{aligned}
$$

Case 5: $\varphi$ is $\psi_{1} \wedge \psi_{2}$. Then

$$
\begin{aligned}
\|\varphi[\vec{a}, \vec{f}]\|^{M} \in G & \Longleftrightarrow\left\|\psi_{1}[\vec{a}, \vec{f}]\right\|^{M} \in G \&\left\|\psi_{2}[\vec{a}, \vec{f}]\right\|^{M} \in G \\
& \Longleftrightarrow M[G] \vDash \psi_{1}\left[\vec{a}, \vec{f}^{G}\right] \& M[G] \vDash \psi_{2}\left[\vec{a}, \vec{f}^{G}\right] \\
& \Longleftrightarrow M[G] \vDash \varphi\left[\vec{a}, \vec{f}^{G}\right] .
\end{aligned}
$$

Case 6: $\varphi$ is $(\exists x) \psi$, where $x$ is a first-order variable. Then

$$
\begin{aligned}
\|\varphi[\vec{a}, \vec{f}]\|^{M} \in G & \Longleftrightarrow \bigvee_{b \in A}\|\psi[b, \vec{a}, \vec{f}]\|^{M} \in G \\
& \Longleftrightarrow(\exists b \in A)\|\psi[b, \vec{a}, \vec{f}]\|^{M} \in G \\
& \Longleftrightarrow(\exists b \in A) M[G] \vDash \psi\left[b, \vec{a}, \vec{f}^{G}\right] \\
& \Longleftrightarrow M[G] \vDash \varphi\left[\vec{a}, \vec{f}^{G}\right] .
\end{aligned}
$$

Case 7: $\varphi$ is $(\exists X) \psi$, where $X$ is a second-order variable. Then

$$
\begin{aligned}
\|\varphi[\vec{a}, \vec{f}]\|^{M} \in G & \Longleftrightarrow \bigvee_{g: A \rightarrow \mathbb{B}}\|\psi[\vec{a}, g, \vec{f}]\|^{M} \in G \\
& \Longleftrightarrow(\exists g: A \rightarrow \mathbb{B})\|\psi[\vec{a}, g, \vec{f}]\|^{M} \in G \\
& \Longleftrightarrow(\exists g: A \rightarrow \mathbb{B}) M[G] \vDash \psi\left[\vec{a}, g^{G}, \vec{f}^{G}\right] \\
& \Longleftrightarrow M[G] \vDash \varphi\left[\vec{a}, \vec{f}^{G}\right] .
\end{aligned}
$$

This completes the proof of the lemma.

We now define one of the most important notions in this paper, the so-called Boolean validity.

Definition 2.5 (Boolean validity) Let $\mathscr{L}$ be as above. Then a second-order $\mathscr{L}$-sentence is Boolean-valid if $\|\varphi\|^{M}=1$ for any Boolean-valued $\mathscr{L}$-structure $M$.

Using Lemma 2.4, one can prove the following useful lemma. 
Lemma 2.6 Let $\mathscr{L}$ be as above. A second-order $\mathscr{L}$-sentence $\varphi$ is Boolean-valid if and only if $\varphi$ is true in any full second-order $\mathscr{L}$-structure in any set-generic extension of $V$.

Proof By Lemma 2.4, the direction from right to left is obvious.

For the direction from left to right, let $\mathbb{B}$ be a complete Boolean algebra in $V$, let $G$ be a $\mathbb{B}$-generic filter over $V$, and let $N=\left(A, \bigcup_{n \in \omega} \mathcal{P}\left(A^{n}\right)^{V[G]},\left\{R_{i}^{N}\right\}_{1 \leq i \leq n}\right)$ be any full second-order $\mathscr{L}$-structure in $V[G]$. We show that $\varphi$ is true in $N$.

First, note that we may assume that $A$ is an ordinal $\gamma$. For each $i$ with $1 \leq i \leq n$, let $m_{i}$ be the natural number such that $R_{i}$ is an $m_{i}$-ary relation symbol, and let $R_{i}^{N}$ be a $\mathbb{B}$-name for $R_{i}^{N}$. Let $M=\left(\gamma, \mathbb{B},\left\{R_{i}^{M}\right\}_{1 \leq i \leq n}\right)$ be the Boolean-valued $\mathscr{L}$-structure, where, for each $\vec{x} \in \gamma^{m_{i}}$,

$$
R_{i}^{M}(\vec{x})=\text { the Boolean value of " } \breve{\vec{x}} \in \dot{R}_{i}^{N} \text { " in } \mathbb{B} .
$$

Then it is easy to show that $M[G]=N$. Hence by Lemma 2.4,

$$
N \vDash \varphi \Longleftrightarrow\|\varphi\|^{M} \in G .
$$

Since $\varphi$ is Boolean-valid, $\|\varphi\|^{M}=1$ and hence $N \vDash \varphi$, as desired.

From the last lemma, it is easy to see that the set of Boolean-valid $\mathscr{L}$-sentences can be seen as a set of natural numbers $\Pi_{2}$-definable in ZFC and therefore, one can reduce this set of natural numbers to the set of Boolean-valid $\mathscr{L}$-sentences of full second-order logic recursively. We will discuss the possibility that the set of Boolean-valid $\mathscr{L}$-sentences can also be seen as a $\Delta_{2}$ set of natural numbers in ZFC later in this article. Also, we will show that Boolean validity is invariant under setforcing extensions assuming a proper class of Woodin cardinals, while the validity of full second-order logic is fragile to set-forcing extensions. For example, one can characterize the set of natural numbers $(\mathbb{N},<)$ and the real line $(\mathbb{R},<)$ (up to isomorphism) with a sentence in full second-order logic, and, using this, one can find a sentence in second-order logic whose validity in full second-order logic is equivalent to the continuum hypothesis, which is not invariant under set-forcing extensions.

We end this section by proving the forcing absoluteness of Boolean validity.

Theorem 2.7 Assume a proper class of Woodin cardinals. Then for any secondorder sentence $\varphi$ and any partial order $\mathbb{P}, \varphi$ is Boolean-valid if and only if $\varphi$ is Boolean-valid in $V^{\mathbb{P}}$.

Proof The statement follows from Theorem 4.2 in Section 4 and Theorem 5.1 in Section 5. Nevertheless, we will give a direct proof using the stationary tower-forcing $\mathbb{P}_{<\delta}$.

Let $\varphi$ be any second-order sentence, and let $\mathbb{P}$ be any partial order. We first show that if $\varphi$ is Boolean-valid, then so is it also in $V^{\mathbb{P}}$. By Lemma 2.6, it suffices to show that $\varphi$ is true in any full second-order structure in any set-generic extension of $V^{\mathbb{P}}$. However, by Lemma 2.6 again, $\varphi$ is true in any full second-order structure in any set-generic extension of $V$, and therefore so is it also in every generic extension of $V^{\mathbb{P}}$.

We finish the proof by showing that if $\varphi$ is Boolean-valid in $V^{\mathbb{P}}$, then so is it also in $V$. We will show the contrapositive; namely, if $\varphi$ is not Boolean-valid in $V$, then it is not Boolean-valid in $V^{\mathbb{P}}$ either. 
Assume that $\varphi$ is not Boolean-valid in $V$. Then by Lemma 2.6, letting $\psi$ be the negation of $\varphi, \psi$ is true in some full second-order structure $N_{0}$ in some generic extension $V^{\mathbb{Q}}$. By Lemma 2.6 again, it is enough to show that $\psi$ is true in some full second-order structure in some generic extension of $V^{\mathbb{P}}$.

Let $\delta$ be a large-enough Woodin cardinal so that $\mathbb{P}, \mathbb{Q}, \dot{N}_{0} \in V_{\delta}$, where $\dot{N}_{0}$ is a $\mathbb{Q}$-name for $N_{0}$. Let $G$ be $\mathbb{P}_{<\delta}$-generic over $V$ such that $\mathcal{P}_{\omega_{1}}(\mathcal{P}(\mathbb{P})) \in G$, and let $j: V \rightarrow M$ be the induced embedding from $G$ with $M^{<\delta} \cap V[G] \subseteq M$. Then in $V[G]$, one can find a $\mathbb{P}$-generic over $V$ and hence $V[G]$ can be seen as a generic extension of $V^{\mathbb{P}}$.

By elementarity of $j, \psi$ is true in the full second-order structure $j\left(\dot{N}_{0}\right)$ in $M^{j(\mathbb{Q})}$. Since $\delta$ is sufficiently large and $M^{<\delta} \cap V[G] \subseteq M$, one can assume that $\psi$ is true in some full second-order structure in $V[G]^{j(\mathbb{Q})}$. Then since $V[G]$ is a generic extension of $V^{\mathbb{P}}, \psi$ is true in some full second-order structure in some generic extension of $V^{\mathbb{P}}$ as well, as desired.

\section{Henkin Models}

We now introduce important structures when discussing second-order logic.

Definition 3.1 (Henkin models) Let $\mathscr{L}=\left\{R_{1}, \ldots, R_{n}\right\}$ be a relational language. A second-order $\mathscr{L}$-structure $M=\left(A, \mathscr{E},\left\{R_{i}^{M}\right\}_{1 \leq i \leq n}\right)$ is a Henkin model if it satisfies the comprehension axiom for each second-order $\mathscr{L}$-formula; that is, for each second-order $\mathscr{L}$-formula $\varphi, \vec{a} \in A^{<\omega}$, and $\vec{f} \in \mathscr{E}^{<\omega}$,

$$
\left\{\vec{x} \in A^{<\omega} \mid M \vDash \varphi(\vec{x}, \vec{a}, \vec{f})\right\} \in \mathcal{E} .
$$

Example 3.2 Let $\mathscr{L}$ be as above. Then a second-order $\mathscr{L}$-structure $M=(A$, $\left.\bigcup_{n \in \omega} \mathcal{P}\left(A^{n}\right),\left\{R_{i}^{M}\right\}_{1 \leq i \leq n}\right)$ is a Henkin model and is called a full second-order $\mathscr{L}$-structure.

The semantics of second-order logic via Henkin models (Henkin semantics) gives us similar logical properties of second-order logic to first-order logic, as in this example.

Theorem 3.3 (Henkin) Second-order logic via Henkin semantics is sound and complete to a standard proof system of second-order logic. In particular, the set of all second-order sentences valid via Henkin semantics is $\Sigma_{1}^{0}$.

On the other hand, the semantics of second-order logic via full second-order structures (full semantics) does not enjoy completeness or compactness; in fact, one can express infinity of a set and well-foundedness of a partial order by second-order sentences via full semantics. Also the validity of second-order logic via full semantics is highly complex.

Theorem 3.4 (Väänänen) Let $\mathscr{L}$ be an arbitrary relational language. Then the set of all second-order valid $\mathscr{L}$-sentences via full semantics is $\Pi_{2}$-complete in the language of set theory.

Proof See [15, Theorem 1].

The Boolean-valued second-order logic is a second-order logic sitting between the two semantics we discussed above. While it is quite powerful (e.g., one can express well-foundedness as well as the theory of Gödel's constructible universe L and some canonical inner models such as $\mathrm{L}^{\mu}$ ), it may enjoy completeness in some sense. 


\section{$4 \Omega$-Logic and $\Omega$-Validity}

We now introduce $\Omega$-logic, especially $\Omega$-validity. $\Omega$-logic is a logic of forcing absoluteness, invented by Hugh Woodin after his invaluable investigation on the connection between forcing absoluteness and large cardinals. Forcing absoluteness discusses preservation of the truth of statements between a ground model and its set-forcing extensions; for example, by Shoenfield's absoluteness lemma, any $\Pi_{2}^{1}$-sentence is forcing-absolute.

Definition 4.1 ( $\Omega$-validity) $\quad \mathrm{A} \Pi_{2}$-sentence $\varphi$ in set theory is $\Omega$-valid if it is true in $V$ and in any set-forcing extension of $V{ }^{1}$

As we mentioned, by Shoenfield's absoluteness lemma, any $\Pi_{2}^{1}$-sentence true in $V$ is $\Omega$-valid. If $V=\mathrm{L}$, the $\Pi_{3}^{1}$-sentence "Every real is constructible" is true, but not $\Omega$-valid, while large cardinals give us more sentences that are $\Omega$-valid. For example, if there is a proper class of Woodin cardinals, then for every sentence $\varphi$ true in $L(\mathbb{R})$, the sentence " $L(\mathbb{R}) \vDash \varphi$ " is $\Omega$-valid (see Larson [4, Corollary 3.1.16]). Furthermore, if there is a proper class of cardinals which are Woodin and measurable, then for any $\Sigma_{1}^{2}$-sentence $\varphi$ true in some set-generic extension, the sentence " $\mathrm{CH} \rightarrow \varphi$ " is $\Omega$-valid (see [4, Theorem 3.2.1]).

It is easy to see that the set of all $\Omega$-valid $\Pi_{2}$-sentences is $\Pi_{2}$ in ZFC. The important point is that $\Omega$-validity is invariant under set-forcing extensions assuming a proper class of Woodin cardinals.

Theorem 4.2 (Woodin) $\quad$ Assume a proper class of Woodin cardinals. Then for any $\Pi_{2}$-sentence $\varphi$ and a set partial order $\mathbb{P}, V \vDash$ " $\varphi$ is $\Omega$-valid" if and only if $V^{\mathbb{P}} \vDash " \varphi$ is $\Omega$-valid."

Proof See, for example, [5, Theorem 2.2].

\section{Boolean Validity and $\Omega$-Validity}

We now prove one of our main results.

Theorem 5.1 Let $\mathscr{L}$ be a relational language containing a binary relation symbol $R$.

(1) There is a recursive translation from second-order $\mathscr{L}$-sentences $\varphi$ to $\Pi_{2}$-sentences $\psi_{\varphi}$ in set theory such that

$$
\varphi \text { is Boolean-valid } \Longleftrightarrow \psi_{\varphi} \text { is } \Omega \text {-valid. }
$$

(2) There is a recursive translation from $\Pi_{2}$-sentences $\psi$ in set theory to secondorder $\mathscr{L}$-sentences $\varphi_{\psi}$ such that

$$
\psi \text { is } \Omega \text {-valid } \Longleftrightarrow \varphi_{\psi} \text { is Boolean-valid. }
$$

In particular, the complexity of Boolean validity is the same as the one of $\Omega$-validity.

Proof We show the first item of the theorem. Given a second-order $\mathscr{L}$-sentence $\varphi$, we define the $\Pi_{2}$-sentence $\psi_{\varphi}$ in set theory stating that

" $\varphi$ is true in any full second-order $\mathscr{L}$-structure." 
We verify the desired equivalence. By Lemma 2.6, $\varphi$ is Boolean-valid if and only if it is true in any full second-order $\mathscr{L}$-structure in any set-generic extension, that is equivalent to that $\psi_{\varphi}$ is $\Omega$-valid, as desired.

Next, we show the second item of the theorem. Given a $\Pi_{2}$-sentence $\psi$ in set theory, we define the second-order $\mathscr{L}$-sentence $\varphi_{\psi}$ stating that

"If $A$ with the relation $R$ is well-founded, extensional, and is isomorphic to $\left(V_{\lambda}, \in\right)$ for some strong limit cardinal $\lambda$, then $(A, R) \vDash \psi$,"

where $A$ is the first-order part of the given structure $M$, and we identify the above $R$ with the interpretation of the binary relation symbol $R$ contained in $\mathscr{L}$. Note that the well-foundedness and the extensionality of a structure $(A, R)$ can be easily expressed by a second-order $\mathscr{L}$-formula. One can also express the statement that " $(A, R)$ is isomorphic to $\left(V_{\lambda}, \in\right)$ for some strong limit cardinal $\lambda$ " via a second-order $\mathscr{L}$-formula by describing a " $V$-hierarchy" function from the set of "ordinals" in $(A, R)$ and the intended " $V_{\alpha}$ " as their images using second-order quantifiers.

We verify the desired equivalence. By Lemma $2.6, \varphi_{\psi}$ is Boolean-valid if and only if it is true in any full second-order $\mathscr{L}$-structure in any set-generic extension, which is equivalent to $V_{\lambda} \vDash \psi$ for all strong limit cardinals $\lambda$ in any set-generic extension because we focus on true power sets in full second-order $\mathscr{L}$-structures in generic extensions. Hence the Boolean validity of $\psi_{\varphi}$ is equivalent to the $\Omega$-validity of $V_{\lambda} \vDash \psi$ for all strong limit cardinals $\lambda$, which is equivalent to the $\Omega$-validity of $\psi$ itself because any $\Sigma_{1}$-formula is absolute between $V_{\lambda}$ for strong limit cardinals $\lambda$ and $V$, as desired.

This completes the proof of our theorem.

The application of the above theorem is discussed in the next section concerning the complexity of Boolean validity and its comparison with that of full second-order logic.

Let us note that Theorem 2.7 would follow from Theorem 4.2 and Theorem 5.1, but we have proved Theorem 2.7 directly to illustrate the method.

\section{$6 \Omega$-Logic, $\Omega$-Provability, and $\Omega$-Conjecture}

We now go back to $\Omega$-logic and discuss $\Omega$-provability and the $\Omega$-conjecture, which were introduced by Hugh Woodin. Considering these notions will help us understand the complexity of Boolean validity.

As mentioned in Section 4, many statements in set theory are $\Omega$-valid, and the more large cardinals we have in $V$, the more statements are $\Omega$-valid. What is behind these phenomena is the notion of universally Baire sets of reals, tying large cardinals with forcing absoluteness, as in the following.

\section{Definition 6.1}

(1) Let $X$ be a topological space, and let $A$ be a subset of $X$.

- The set $A$ is nowhere dense in $X$ if it is disjoint from a dense open subset of $X$.

- The set $A$ is meager in $X$ if it is a countable union of nowhere-dense sets in $X$.

- The set $A$ has the Baire property if there is an open set $U$ in $X$ such that the symmetric difference between $A$ and $U$ is meager. 
(2) A set of reals $A$ is universally Baire if, for any compact Hausdorff space $X$ and any continuous function $f: X \rightarrow \mathbb{R}$, the set $f^{-1}(A)$ has the Baire property in $X$.

The more large cardinals we have, the more complicated sets of reals become universally Baire and the more complicated statements become $\Omega$-valid. In fact, any transitive model of ZFC closed under certain universally Baire sets is "correct" about the truth of certain statements. $\Omega$-provability is the notion capturing this idea.

\section{Definition 6.2}

(1) Let $A$ be a universally Baire set of reals, and let $M$ be a countable transitive model of ZFC. We say that $M$ is strongly $A$-closed ${ }^{2}$ if, for any partial order $\mathbb{P}$ in $M$ and any $\mathbb{P}$-generic filter $G$ over $M$,

$$
A \cap M[G] \in M[G] .
$$

(2) Let $\varphi$ be a $\Pi_{2}$-sentence. We say that $\varphi$ is $\Omega$-provable if there is a universally Baire set of reals $A$ such that for any countable transitive model $M$ of ZFC, if $M$ is strongly $A$-closed, then $M \vDash \varphi .^{3}$

One can define strong $A$-closure even for countable $\omega$-models of ZFC (instead of countable transitive models of ZFC) and an $\omega$-model $M$ of ZFC is strongly $A$-closed for any $\Pi_{1}^{1}$ (lightface) set $A$ if and only if $M$ is well-founded. Therefore, strong $A$-closure is a generalization of well-foundedness.

Let us give more examples of strong $A$-closure and $\Omega$-provable sentences. Suppose every set has a sharp. Then the set $A=\left\{(x, n) \in \mathbb{R} \times \omega \mid n \in x^{\#}\right\}$ is universally Baire and $M$ is strongly $A$-closed if and only if $M$ is closed under sharps. Since any transitive model of ZFC closed under sharps is correct about the truth of $\Sigma_{2}^{1}$-formulas, every $\Pi_{3}^{1}$-sentence true in $V$ is $\Omega$-provable assuming that every set has a sharp. In the same way, if $M_{\omega}^{\#}(X)$ exists for any set $X$, then for any sentence $\varphi$ true in $\mathrm{L}(\mathbb{R})$, the sentence " $\mathrm{L}(\mathbb{R}) \vDash \varphi$ " is $\Omega$-provable by looking at the strong closure of the set $A=\left\{(x, n) \in \mathbb{R} \times \omega \mid n \in M_{\omega}^{\#}(x)\right\}$, where $M_{\omega}^{\#}(X)$ is the minimal $X$-mouse with $\omega$-many Woodin cardinals and a top measure. For the details of this notion, one can refer to Steel [13].

The soundness holds for $\Omega$-logic, as in the following.

Theorem 6.3 (Woodin) Let $\varphi$ be a $\Pi_{2}$ sentence in set theory. If $\varphi$ is $\Omega$-provable, then it is $\Omega$-valid.

Proof See, for example, [5, Theorem 6.4].

Woodin's $\Omega$-conjecture states that $\Omega$-provability and $\Omega$-validity are the same thing, as in this conjecture.

Conjecture 6.4 (Woodin) $\quad$ The $\Omega$-conjecture is the following statement:

Suppose there is a proper class of Woodin cardinals. Then for any $\Pi_{2}$-sentence $\varphi$ in set theory, $\varphi$ is $\Omega$-provable if and only if $\varphi$ is $\Omega$-valid.

It is not known whether one can prove the $\Omega$-conjecture while Woodin showed that the $\Omega$-conjecture is consistent, as follows.

Theorem 6.5 (Woodin) $\quad$ Assume a proper class of Woodin cardinals. Then there is a transitive model of ZFC with a proper class of Woodin cardinals in which the $\Omega$-conjecture is true. 
Proof See, for example, [5, Section 9.2].

Moreover, Woodin showed that $\Omega$-provability is invariant under set-forcing extensions assuming a proper class of Woodin cardinals (see [1, Theorem 2.35]). Combining it with Theorem 4.2 , one can see that the $\Omega$-conjecture is invariant under set-forcing extension as well.

One great consequence of the $\Omega$-conjecture is a finer analysis of the complexity of $\Omega$-validity by the following result of Woodin.

Theorem 6.6 (Woodin) $\quad$ Assume a proper class of Woodin cardinals, and let $\delta$ be the first Woodin cardinal. Then the set of $\Omega$-provable $\Pi_{2}$-sentences in set theory is definable in $\mathscr{H}_{\delta}+$.

Proof See, for example, [5, Section 8.1].

Corollary 6.7 Assume a proper class of Woodin cardinals, and suppose that the $\Omega$-conjecture is true. Then the set of all $\Omega$-valid $\Pi_{2}$-sentences in set theory is $\Delta_{2}$ in ZFC.

Combining the above corollary with Theorem 5.1, one can get this interesting result on Boolean-valued second-order logic.

Corollary 6.8 Assume a proper class of Woodin cardinals, and suppose that the $\Omega$-conjecture is true. Let $\mathscr{L}$ be a relational language. Then the set of Boolean-valid $\mathscr{L}$-sentences is $\Delta_{2}$ in ZFC.

The result is in contrast with the fact that the complexity of the set of valid $\mathscr{L}$-sentences in full second-order logic is $\Pi_{2}$-complete in ZFC (see Theorem 3.4).

\section{Boolean-Valued Second-Order Logic, Boolean Provability}

After reviewing $\Omega$-provability, we now introduce Boolean provability in Booleanvalued second-order logic as an analogue of $\Omega$-provability, and we investigate the connection between Boolean provability and Boolean validity. We start with introducing forcing in Henkin models.

Definition 7.1 (Generic extensions) Let $\mathscr{L}=\left\{R_{1}, \ldots, R_{n}\right\}$ be a relational language, and let $M=\left(A, \mathcal{E},\left\{R_{i}^{M}\right\}_{1 \leq i \leq n}\right)$ be a Henkin model. Let $\mathbb{B} \in \mathcal{E}$, meaning $\mathbb{B}=\left(B, \wedge_{\mathbb{B}}, \vee_{\mathbb{B}}, 0_{\mathbb{B}}, 1_{\mathbb{B}}\right)$, where $B, \wedge_{\mathbb{B}}, \vee_{\mathbb{B}} \in \mathcal{G}$ and $0_{\mathbb{B}}, 1_{\mathbb{B}} \in A$, and let $\mathbb{B}$ be a complete Boolean algebra in $M$, that is, the second-order sentence that $\mathbb{B}$ is a complete Boolean algebra is true in $M$. Given a $\mathbb{B}$-generic filter $G$ over $M$, one can define a second-order $\mathscr{L}$-structure $M[G]=\left(B, \mathscr{H},\left\{R_{i}^{M[G]}\right\}_{1 \leq i \leq n}\right)$ as follows:

(1) $B=A$ and $R_{i}^{M[G]}=R_{i}^{M}$ for each $i$ with $1 \leq i \leq n$,

(2) $\mathscr{H}=\left\{f^{G} \mid f \in \mathbb{B}^{A^{<\omega}} \cap \mathcal{E}\right\}$, where $f^{G}=\left\{\vec{x} \in A^{<\omega} \mid f(\vec{x}) \in G\right\}$.

Definition 7.2 Given $\mathscr{L}, M, \mathbb{B}$ as above, we assign the Boolean value $[\varphi[\vec{a}, \vec{f}]]^{M}$ (an element of $\mathbb{B}$ ) to each second-order $\mathscr{L}$-formula $\varphi, \vec{a} \in A^{<\omega}, \vec{f} \in\left(\mathbb{B}^{A} \cap \mathcal{E}\right)^{<\omega}$ as follows:

(1) $\varphi$ is $x=y$, where $x, y$ are first-order variables; then $[x=y[a, b]]^{M}$ is 1 if $a=b$ and 0 otherwise.

(2) $\varphi$ is $R_{i}(x)$, where $x$ is a first-order variable; then $\left[R_{i}[a]\right]^{M}=R_{i}^{M}(a)$. 
(3) $\varphi$ is $X(x)$, where $X$ is a second-order variable and $x$ is a first-order variable; then $[X(x)[a, f]]^{M}=f(a)$.

(4) $\varphi$ is $\neg \psi$; then $[\varphi[\vec{a}, \vec{f}]]^{M}=1-[\psi[\vec{a}, \vec{f}]]^{M}$.

(5) $\varphi$ is $\psi_{1} \wedge \psi_{2}$; then $[\varphi[\vec{a}, \vec{f}]]^{M}=\left[\psi_{1}[\vec{a}, \vec{f}]\right]^{M} \wedge\left[\psi_{2}[\vec{a}, \vec{f}]\right]^{M}$.

(6) $\varphi$ is $(\exists x) \psi$, where $x$ is a first-order variable; then

$$
[\varphi[\vec{a}, \vec{f}]]^{M}=\bigvee_{b \in A}[\psi[b, \vec{a}, \vec{f}]]^{M} .
$$

(7) $\varphi$ is $(\exists X) \psi$, where $X$ is a second-order variable; then

$$
[\varphi[\vec{a}, \vec{f}]]^{M}=\bigvee_{g \in\left(\mathbb{B}^{A} \cap \mathcal{E}\right)}[\psi[\vec{a}, g, \vec{f}]]^{M} .
$$

Note that the above definition is the same as Definition 2.2 except for the last clause. In the last clause, we take the supremes over all the functions in $\mathcal{G}$ instead of all the functions in $V$.

We now show that one can proceed as with the usual forcing method within Henkin models.

Theorem 7.3 Let $\mathscr{L}, M, \mathbb{B}$ be as above, and let $G$ be a $\mathbb{B}$-generic filter over $M$.

(1) For each second-order $\mathscr{L}$-formula $\varphi, \vec{a} \in A^{<\omega}$, and $\vec{f} \in\left(\mathbb{B}^{A} \cap \mathcal{E}\right)^{<\omega}$,

$$
M[G] \vDash \varphi\left[\vec{a}, \vec{f}^{G}\right] \Longleftrightarrow[\varphi[\vec{a}, \vec{f}]]^{M} \in G .
$$

(2) $M[G]$ is a Henkin model.

Proof For the first item, the argument is the same as in the proof of Lemma 2.4.

For the second item, let $\varphi$ be any second-order $\mathscr{L}$-formula, $\vec{a} \in A^{<\omega}$, and $\vec{f} \in\left(\mathbb{B}^{A} \cap \mathcal{E}\right)^{<\omega}$. It is enough to show that the set

$$
A=\left\{\vec{x} \in A \mid M[G] \vDash \varphi\left[\vec{x}, \vec{a}, \vec{f}^{G}\right]\right\}
$$

belongs to $\mathscr{H}$, where $\mathscr{H}=\left\{g^{G} \mid g \in \mathbb{B}^{A^{<\omega}} \cap \mathcal{E}\right\}$. For simplicity, let $\vec{x}$ be one variable $x$, and let $g: A \rightarrow \mathbb{B}$ be as follows:

$$
g(x)=[\varphi[x, \vec{a}, \vec{f}]]^{M} .
$$

Then since $M$ is a Henkin model, $g$ is in $\mathcal{E}$. It suffices to show that $g^{G}=A$, but by the first item of this theorem, for each $x \in A$,

$$
\begin{aligned}
x \in A & \Longleftrightarrow[\varphi[x, \vec{a}, \vec{f}]]^{M} \in G \\
& \Longleftrightarrow g(x) \in G \\
& \Longleftrightarrow x \in g^{G} .
\end{aligned}
$$

Hence $g^{G}=A$, as desired.

We now introduce the Boolean provability in Boolean-valued second-order logic as an analogy of $\Omega$-provability in $\Omega$-logic. In the definition of $\Omega$-provability, we used countable transitive models of $\mathrm{ZFC}$ as "test models." We will introduce adequate Henkin models for the counterpart of transitive models of ZFC in the context of Boolean-valued second-order logic. 
Definition 7.4 (Adequate Henkin models) Let $\mathscr{L}=\left\{R_{i}\right\}_{1 \leq i \leq n}$ be a relational language. Let $M=\left(A, \mathscr{G},\left\{R_{i}^{M}\right\}_{1 \leq i \leq n}\right)$ be a second-order $\mathscr{L}$-structure which is a Henkin model.

(1) We say that $M$ is an $\omega$-model if $M$ thinks " $A$ is infinite" (i.e., $(\exists x \in A$ ) $(\exists f \in \mathcal{E})), f$ is a bijection between $A$ and $A \backslash\{x\})$, and $\left(\omega^{M},<^{M}\right)$ is isomorphic to $(\omega,<)$ in $V$, where $\left(\omega^{M},<^{M}\right)$ is a unique structure satisfying the axioms for natural numbers. ${ }^{4}$

(2) We say that $M$ is adequate if

- $M$ is an $\omega$-model,

- there is a pairing function of $A$ (i.e., a bijection between $A$ and $A^{2}$ ) which is in $\mathcal{E}$, and

- there is a well-order on $A$ in $\mathcal{E}$.

We now define the Boolean provability in Boolean-valued second-order logic. Let us write $\mathbb{R}$ for $\mathcal{P}(\omega)$; we continue to use this convention for the rest of this article.

Definition 7.5 (Boolean provability) Let $\mathscr{L}$ be as above.

(1) Let $f: \mathbb{R} \rightarrow \mathbb{R}$ be universally Baire (i.e., its graph is a universally Baire subset of $\left.\mathbb{R}^{2}\right)$, and let $M=\left(A, \mathcal{E},\left\{R_{i}\right\}_{1 \leq i \leq n}\right)$ be a second-order $\mathscr{L}$-structure which is a countable adequate Henkin model. We say that $M$ is $f$-closed if, for any $\mathbb{B} \in \mathscr{E}$ which is a complete Boolean algebra in $M$ and any $\mathbb{B}$-generic filter $G$ over $M, M[G]$ is closed under $f$; that is, for any real $x \subseteq \omega$ such that $\rho(x) \in M[G], \rho(f(x)) \in M[G]$, where $\rho:(\omega,<) \rightarrow\left(\omega^{M},<^{M}\right)$ is an isomorphism. ${ }^{5}$

(2) Let $\varphi$ be a second-order $\mathscr{L}$-sentence. We say that $\varphi$ is Boolean-provable if there is a function $f: \mathbb{R} \rightarrow \mathbb{R}$ which is universally Baire such that if $M$ is an $f$-closed countable adequate Henkin model, then $M \vDash$ " $\varphi$ is Boolean-valid."

We now discuss the connection between Boolean provability and Boolean validity.

Theorem 7.6 (Soundness) Let $\mathscr{L}$ be as above, and let $\varphi$ be a second-order $\mathscr{L}$-sentence. If $\varphi$ is Boolean-provable, then $\varphi$ is Boolean-valid.

Proof Assume that $\varphi$ is Boolean-provable, and let $f: \mathbb{R} \rightarrow \mathbb{R}$ be its witness. Let $\lambda$ be a strong limit cardinal such that the statement " $\varphi$ is Boolean-valid" is absolute between $V_{\lambda}$ and $V$, and such that $f \in V_{\lambda}$ and $V_{\lambda} \vDash$ " $f$ is universally Baire." It suffices to show that $\varphi$ is Boolean-valid in $V_{\lambda}$.

Let $X$ be a countable elementary submodel of $V_{\lambda}$ containing $f$, and let $N$ be its transitive collapse. Then $N$ is strongly $A$-closed where $A=\{(x, n) \in \mathbb{R} \times \omega \mid n \in$ $f(x)\} .{ }^{6}$ Since $X$ is an elementary submodel of $V_{\lambda}$, it is enough to show that $N \vDash \varphi$ is Boolean-valid."

Let $M$ be a Boolean-valued $\mathscr{L}$-structure in $N$, and let $\alpha$ be a limit ordinal in $N$ such that $M \in V_{\alpha}^{N}$. Let $N_{0}=\left(V_{\alpha}^{N}, V_{\alpha+1}^{N},\left\{R_{i}^{N_{0}}\right\}\right)$, where $R_{i}^{N_{0}}=\emptyset$ for each $i$ with $1 \leq i \leq n$. Then $N_{0}$ is a countable adequate Henkin model.

We first show that $N_{0}$ is $f$-closed, and then we show that $\|\varphi\|^{M}=1$ in $N$. To see that $N_{0}$ is $f$-closed, let us take any complete Boolean algebra $\mathbb{B}$ in $N_{0}$ and any $\mathbb{B}$-generic filter $G$ over $N_{0}$. We will show that $N_{0}[G]$ is closed under $f$. First note that $G$ is also $\mathbb{B}$-generic over $N$ since all the dense subsets of $\mathbb{B}$ in $N$ belong to $V_{\alpha+1}^{N}$. Therefore, by the strong $A$-closure of $N$ where $A=\{(x, n) \in \mathbb{R} \times \omega \mid n \in f(x)\}$, $N[G]$ is closed under $f$. But then $N_{0}[G]$ is also closed under $f$ since any real in $N[G]$ can be coded by $G$ and a $\mathbb{B}$-name in $V_{\alpha+1}^{N}$. Thus, $N_{0}$ is $f$-closed. 
We finish the proof by showing that $\|\varphi\|^{M}=1$ in $N$. Since $N_{0}$ is $f$-closed and $\varphi$ is Boolean-provable via $f, N_{0}$ believes that $\varphi$ is Boolean-valid. Hence $\|\varphi\|^{M}=1$ in $N_{0}$, and therefore $\|\varphi\|^{M}=1$ is also true in $N$ because $N_{0}$ was a large rank initial segment of $N$, as desired. Since $M$ was arbitrary in $N, \varphi$ is Boolean-valid in $N$ and hence in $V_{\lambda}$ and in $V$. Therefore, $\varphi$ is Boolean-valid.

Definition 7.7 (Completeness) The completeness of Boolean-valued secondorder logic is the following.

Let $\mathscr{L}=\left\{R_{0}, \ldots, R_{n}\right\}$ be a finite relational language, and let $\varphi$ be any secondorder $\mathscr{L}$-sentence. Then if $\varphi$ is Boolean-valid, $\varphi$ is Boolean-provable.

The completeness of Boolean-valued second-order logic is not known to be consistent. In the next section, we compare this completeness with the $\Omega$-conjecture.

\section{The $\Omega$-Conjecture and Completeness of Boolean-Valued Second-Order Logic}

We now state one of our main results about Boolean validity.

Theorem 8.1 The completeness of Boolean-valued second-order logic implies the $\Omega$-conjecture.

Proof Let $\psi$ be a $\Pi_{2}$-sentence in the language of set theory which is $\Omega$-valid. We show that $\psi$ is $\Omega$-provable assuming the completeness of Boolean-valued secondorder logic. Let $\mathscr{L}=\{R\}$ with a binary relation symbol $R$, and define the secondorder $\mathscr{L}$-sentence $\varphi_{\psi}$ as in the proof of Theorem 5.1, that is,

"If $A$ with the relation $R$ is well-founded, extensional, and is isomorphic to $\left(V_{\lambda}, \in\right)$ for some strong limit cardinal $\lambda$, then $(A, R) \vDash \psi$,"

where $A$ is the first-order part of the given second-order structure $M$, and identify the above $R$ with the interpretation of the binary relation symbol $R$ in $\mathscr{L}$.

By Theorem 5.1, $\varphi_{\psi}$ is Boolean-valid. Therefore, by the completeness of Boolean-valued second-order logic, $\varphi_{\psi}$ is Boolean-provable. Let $f: \mathbb{R} \rightarrow \mathbb{R}$ witness the Boolean provability of $\varphi_{\psi}$, and let $A=\{(x, n) \in \mathbb{R} \times \omega \mid n \in f(x)\}$. Then $A$ is universally Baire, and we show that $A$ witnesses that $\psi$ is $\Omega$-provable.

Let $N$ be a countable transitive model of ZFC which is strongly $A$-closed. We show that $\psi$ is true in $N$. Since $\psi$ is $\Pi_{2}$, if suffices to show that $V_{\alpha}^{N} \vDash \psi$ for each strong limit cardinal $\alpha$ in $N$.

Take an arbitrary strong limit cardinal $\alpha$ in $N$, and consider the second-order $\mathscr{L}$-structure $M=\left(V_{\alpha}^{N}, V_{\alpha+1}^{N}, \in \cap\left(V_{\alpha}^{N} \times V_{\alpha}^{N}\right)\right)$. Then $M$ is a countable adequate Henkin model. By the same argument given in the proof of Theorem 7.6, $M$ is $f$-closed, and hence $\varphi_{\psi}$ is Boolean-valid in $M$, and in particular this is true in $M$. By the definition of $\varphi_{\psi}, V_{\alpha}^{N} \vDash \psi$. Since $\alpha$ was an arbitrary strong limit cardinal in $N, \psi$ is true in $N$, as desired.

The main open problem is the following.

Question 8.2 Is the completeness of Boolean-valued second-order logic equivalent to the $\Omega$-conjecture assuming the existence of a proper class of Woodin cardinals?

The following question is, of course, of most importance but still open. 
Question 8.3 Is the completeness of Boolean-valued second-order logic consistent?

Question 8.4 Are there any other natural provability notions for Boolean-valued second-order logic which would be consistent?

\section{Cardinal Characteristics of Boolean-Valued Second-Order Logic}

In this section, we consider the Hanf number as well as the numbers related to the compactness and the Löwenheim number of Boolean-valued second-order logic, and we show that they are strictly smaller (or consistently smaller) than those of the full second-order logic, under some assumptions of large cardinals and $\Omega$-logic.

We now discuss the cardinals related to compactness in some extensions of Boolean-valued second-order logic and full second-order logic.

For regular cardinals $\kappa, \lambda$, let $\mathrm{L}_{\kappa, \lambda}^{2}$ denote the second-order logic with $<\kappa$-long conjunctions and disjunctions and $<\lambda$-long sequences of (first-order and secondorder) quantifiers when forming the set of formulas in this logic. The semantics of these formulas is defined in a standard way.

Respectively, for regular cardinals $\kappa, \lambda$, let $\mathrm{L}_{\kappa, \lambda}^{2 b}$ denote the Boolean-valued second-order logic with $<\kappa$-long conjunctions and disjunctions and $<\lambda$-long sequences of quantifiers when forming the set of formulas in this logic. The semantics of these formulas is the same as that of formulas in $\mathrm{L}_{\kappa, \lambda}^{2}$ except that of the secondorder quantifiers. The semantics of the second-order quantifiers (or sequences of second-order quantifiers) is analogously given as in the definition of the semantics of Boolean-valued second-order logic.

For a given logic $\mathrm{L}^{*}$ and an uncountable regular cardinal $\kappa, \kappa$ is called $\mathrm{L}^{*}$-compact if for any set of sentences $T$ in $\mathrm{L}^{*}$, if any subset of $T$ with cardinality less than $\kappa$ has a model, then $T$ also has a model.

The following is a result due to Magidor on the compactness of $\mathrm{L}_{\kappa, \kappa}^{2}$ related to large cardinals.

Theorem 9.1 (Magidor) Let $\kappa$ be a regular uncountable cardinal. Then the following are equivalent:

(1) $\kappa$ is extendible,

(2) $\kappa$ is $\mathrm{L}_{\kappa, \kappa}^{2}$-compact, and

(3) $\kappa$ is $\mathrm{L}_{\kappa, \omega}^{2}$-compact.

\section{Proof See [6].}

In contrast, we show that supercompactness of $\kappa$ is enough to ensure the compactness of $\mathrm{L}_{\kappa, \kappa}^{2 b}$ under some assumptions on large cardinals and $\Omega$-logic.

Note that for a real $x$ and a $\Pi_{2}$-formula $\varphi(z)$ in the language of set theory, one can define the $\Omega$-validity and $\Omega$-provability of the formula $\varphi(x)$, where $x$ is a parameter, in the same way as those of $\Pi_{2}$-sentences. (For $\Omega$-provability, one can restrict the strongly $A$-closed models with those containing $x$ as an element in its definition.) Note also that the soundness for the statements of the form $\varphi(x)$ where $\varphi$ is a $\Pi_{2}$-formula and $x$ is a real, holds. 


\section{Definition 9.2 (Strong $\boldsymbol{\Omega}$-conjecture)}

(1) The $\Omega$-conjecture with real parameters states the following. Assume a proper class of Woodin cardinals. Then for each $\Pi_{2}$-formula $\varphi$ in set theory and any real $x, \varphi(x)$ is $\Omega$-provable if and only if $\varphi(x)$ is $\Omega$-valid.

(2) The strong $\Omega$-conjecture states that the $\Omega$-conjecture with real parameters holds in any set-forcing extension.

We will prove that the strong $\Omega$-conjecture is consistent with a proper class of Woodin cardinals. For the proof, we use the notion of extenders and iteration trees. For the basics of those notions, one can refer to Martin and Steel [7]. Beside the basic notions of extenders and iteration trees that appeared in [7], we will use the following notions.

\section{Definition 9.3}

(1) An extender $E$ is short if $\operatorname{lh}(E) \leq i_{E}(\operatorname{crit}(E))$, where $\operatorname{lh}(E)$ is the length of $E, i_{E}$ is the ultrapower embedding induced by $E$, and $\operatorname{crit}(E)$ is the critical point of $i_{E}$.

(2) An extender $E$ is nice if it is short, $\operatorname{lh}(E)=\operatorname{strength}(E)$, and $\operatorname{lh}(E)$ is inaccessible, where strength $(E)$ is the strength of $E$.

(3) An iteration tree $\mathcal{T}=\left\langle T,\left(E_{\alpha} \mid \alpha+1<\theta\right)\right\rangle$ is normal if there are ordinals $\left(\rho_{\alpha} \mid \alpha+1<\theta\right)$ such that for all ordinals $\alpha, \beta$ with $\alpha+1, \beta+1<\theta$,

(a) $\rho_{\alpha}+2 \leq \operatorname{strength}\left(E_{\alpha}^{\mathcal{T}}\right)$,

(b) $\rho_{\alpha}<\rho_{\beta}$, and

(c) if $\alpha$ is the immediate $T$-predecessor of $\beta+1$, then $\alpha$ is the least $\gamma$ such that $\operatorname{crit}\left(E_{\beta}\right) \leq \rho_{\gamma}$.

(4) An iteration tree $\left.\mathcal{T}=\left\langle T,\left(E_{\alpha} \mid \alpha+1<\theta\right)\right\rangle\right)$ is nice if it is normal and $M_{\alpha}^{\mathcal{T}} \vDash " E_{\alpha}$ is nice" for each $\alpha<\theta$, where $M_{\alpha}^{\mathcal{T}}$ is the $\alpha$ th model of the iteration tree $\mathcal{T}$.

We now introduce the key hypothesis when discussing the strong $\Omega$-conjecture.

Definition 9.4 Nice unique branch hypothesis (Nice-UBH) is the following statement.

Suppose that $\mathcal{T}$ is a countable and nice iteration tree on $V$ of limit length. Then $\mathcal{T}$ has at most one cofinal well-founded branch.

Theorem 9.5 (Woodin) The theory $Z F C+$ strong $\Omega$-conjecture + "There is a proper class of Woodin cardinals" is consistent assuming the consistency of the theory ZFC + "There is a proper class of Woodin cardinals."

Proof We will use some notions from inner model theory. For the notions such as "mouse," "fully iterable," and "L[$\vec{E}]$," one can refer to Mitchell and Steel [8] and Steel [13].

The statement follows from the following three theorems.

Theorem 9.6 (Mitchell, Steel) Assume a proper class of Woodin cardinals. Then there is a fully iterable proper class mouse $\mathrm{L}[\vec{E}]$ with a proper class of Woodin cardinals.

Theorem 9.7 (Schlutzenberg, Steel) Suppose that $V=\mathrm{L}[\vec{E}]$ and that it is fully iterable. Then nice-UBH is true. 
Theorem 9.8 (Woodin) Assume a proper class of Woodin cardinals. Then nice-UBH implies the strong $\Omega$-conjecture.

Proof of Theorem 9.6 See [8] and [13].

Proof of Theorem 9.7 Suppose that $\mathcal{T}$ is a countable and nice iteration tree on $V$ of limit length. We will show that $\mathcal{T}$ has at most one cofinal well-founded branch.

Since $\mathcal{T}$ is nice and $\mathrm{L}[\vec{E}]$ is fully iterable, by Schlutzenberg [11, Corollary 2.10], each extender in $\mathcal{T}$ is in the sequence $\vec{E}$. On the other hand, by Steel [12, Theorem 3.3], every countable and nice iteration tree on $V=\mathrm{L}[\vec{E}]$ has at most one cofinal well-founded branch if every extender on the tree is from its sequence. Therefore, $\mathcal{T}$ has at most one cofinal well-founded branch.

Proof of Theorem 9.8 We will use the following key lemma.

Lemma 9.9 Assume a Woodin cardinal $\delta$, an inaccessible cardinal $\kappa>\delta$, and $a$ set of extenders $\mathbb{E} \subseteq V_{\delta}$ such that

(1) E witnesses that $\delta$ is Woodin,

(2) each extender in $\&$ is nice.

Assume a countable elementary substructure $X$ of $\left(V_{\kappa}, \in\right)$ with $\& \in X$ such that if $M_{X}, \overline{\mathcal{E}}$ are the images of $X$ and $\mathcal{E}$ via the transitive collapse, then $M_{X}$ has an $\omega_{1}$-iteration strategy for nice iteration trees whose extenders are from $\overline{\mathcal{E}}$ which is universally Baire in the codes (i.e., the set of reals coding elements of the $\omega_{1}$-iteration strategy is universally Baire).

Then the $\Omega$-conjecture with real parameters holds.

Proof See, for example, [5, Section 9.2].

Let $\mathbb{P}$ be a partial order and let $G$ be $\mathbb{P}$-generic over $V$. We show that the $\Omega$-conjecture with real parameters holds in $V[G]$. By Lemma 9.9, it is enough to find $\delta<\kappa$ and $\varepsilon$ with the properties described in Lemma 9.9 in $V[G]$.

In $V$, let $\delta_{0}$ be a limit of Woodin cardinals such that $\mathbb{P} \in V_{\delta_{0}}$ and such that in $V[G]$, every $<\delta_{0}$-universally Baire set is universally Baire. Let $\delta>\delta_{0}$ be a Woodin cardinal in $V$. Then $\delta$ is Woodin in $V[G]$ as well. Let $\varepsilon \subseteq V[G]_{\delta}$ be a set of nice extenders with critical points above $\delta_{0}$ such that $\mathcal{E}$ witnesses that $\delta$ is Woodin in $V[G]$. Let $\kappa>\delta$ be inaccessible in $V[G]$.

Claim $1 V[G]_{\kappa}$ satisfies nice-UBH for nice iteration trees whose extenders are induced by those from $\mathcal{E}$.

Proof of Claim $1 \quad$ Let $\mathcal{T}$ be a countable and nice iteration tree on $V[G]_{\kappa}$ of limit length whose extenders are induced by those from $\mathcal{E}$. We will show that there is at most one cofinal well-founded branch of $\mathcal{T}$.

We will use nice-UBH in $V$. Let $\mathcal{T}$ be as in the statement, and suppose that there are two cofinal well-founded distinct branches $b, c$ of $\mathcal{T}$. Then since $\mathbb{P}$ is in $V_{\delta_{0}}$ and $\delta_{0}$ is a limit of Woodin cardinals, there is a Woodin cardinal $\delta^{\prime}<\delta_{0}$ such that $\mathbb{P} \in V_{\delta^{\prime}}$. So one can embed $\mathbb{P}$ into the stationary tower forcing $\mathbb{Q}<\delta^{\prime}$ below some condition and induce the corresponding iteration tree $\mathcal{T}^{\prime}$ and its branches $b^{\prime}, c^{\prime}$ in $V^{\mathbb{Q}<\delta_{0}}$ with the same properties. So we may assume that $\mathbb{P}=\mathbb{Q}<\delta^{\prime}$.

Let $G$ be $\mathbb{Q}<\delta^{\prime}$-generic over $V$, and let $j: V \rightarrow M$ be the induced embedding from $G$. Since every extender in $\varepsilon$ has critical points above $\delta_{0}$, for any extender $E$ 
in $\mathcal{T}, E \cap V$ is in $V$ and one can consider sending these extenders via $j$ and forming a corresponding iteration tree to $\mathcal{E}$ in $M$. Let us call this iteration tree $j(\mathcal{E})$ for the ease of notation.

The point is that for each $E \in \mathcal{E}, i_{E}^{V[G]} \uparrow M=i_{j(E \cap V)}^{M}$. This is done by the same argument as in Woodin [18, Lemma 147]. Hence the iteration tree $j(\mathcal{T})$ in $M$ has two cofinal well-founded branches $j^{\prime \prime} b$ and $j^{\prime \prime} c$ in $M$, contradicting nice-UBH in $V$ and the elementarity of $j: V \rightarrow M$.

Now it is enough to prove the assumption of Lemma 9.9 in $V[G]$ (i.e., there is a countable elementary substructure $X$ of $\left(V[G]_{\kappa}, \in\right)$ with $\varepsilon \in X$ such that if $M_{X}, \bar{\varepsilon}$ are the images of $X$ and $\mathcal{E}$ via the transitive collapse, then $M_{X}$ has a universally Baire $\omega_{1}$-iteration strategy for nice iteration trees whose extenders are from $\bar{\varepsilon}$ ).

However, this holds for any countable elementary substructure $X$ with $\& \in X$ as we shall see below. Let $\pi: M_{X} \rightarrow V[G]_{\kappa}$ be the inverse of the transitive collapse of $X$. We will define the desired $\omega_{1}$-iteration strategy $\Sigma$ as follows. Given a countable and nice iteration tree $\mathcal{T}$ with limit length whose extenders are from $\overline{\mathcal{E}}$, let $\Sigma(\mathcal{T})$ be a unique $\pi$-realizable branch (i.e., a unique branch $b$ such that $\pi^{\prime \prime} b$ is the unique cofinal well-founded branch of $\pi_{*} \mathcal{T}$ in $V[G]_{\kappa}$, where $\pi_{*} \mathcal{T}$ is the iteration tree induced from $\pi$ and $\mathcal{T}$ ). This is well defined since nice-UBH for relevant iteration trees holds in $V[G]_{\kappa}$ by Claim 1 .

We show that $\Sigma$ is an $\omega_{1}$-iteration strategy of $M_{X}$ which is universally Baire in the codes. Being an $\omega_{1}$-iteration strategy follows from nice-UBH in $V[G]_{\kappa}$. Using nice-UBH and the tree production lemma (see, e.g., [4, Theorem 3.3.15]), one can show it is universally Baire in the codes. To see that, there are two points:

(a) every $<\delta_{0}$-universally Baire set is universally Baire in $V[G]$ and the critical points of extenders from $\mathcal{E}$ are above $\delta_{0}$;

(b) for any Woodin $\delta^{\prime}<\delta_{0}$ in $V[G]$, if $H \subseteq \mathbb{Q}_{<\delta^{\prime}}$ is $V[G]$-generic and $j: V[G] \rightarrow M$ is from $H$, then for each extender $E$ from $\&, i_{E}^{V[G, H]} \uparrow M=$ $i_{j(E)}^{M}$.

Item (a) follows from the choice of $\delta_{0}$ and $\mathcal{E}$. Item (b) is proved in [18, Lemma 147]. The rest is a standard argument to prove $\Sigma$ is universally Baire in the codes.

Theorem 9.10 Assume the existence of a proper class of Woodin cardinals and that the strong $\Omega$-conjecture holds. If $\kappa$ is a supercompact cardinal, then $\kappa$ is $\mathrm{L}_{\kappa, \kappa}^{2 b}$-compact.

Note that if $\kappa$ is $\mathrm{L}_{\kappa, \kappa}^{2 b}$-compact, then $\kappa$ must be strongly compact because the compactness of infinitary Boolean-valued second-order logic implies that of infinitary first-order logic.

Proof Let $T$ be a set of sentences in $\mathrm{L}_{\kappa, \kappa}^{2 b}$ such that any subset of $T$ with cardinality less than $\kappa$ has a Boolean-valued model. We show that $T$ itself has a Boolean-valued model.

Let $\eta>\kappa$ be a limit of Woodin cardinals such that $T \in V_{\eta}$. Since $\kappa$ is supercompact, there is an elementary embedding $j: V \rightarrow M$ with critical point $\kappa$ such that $j(\kappa)>\eta, j \uparrow V_{\eta} \in M$, and $M$ is transitive. 
The outline to have a Boolean-valued model of $T$ in $V$ is as follows. By the conditions of $j$, one can see $j^{\prime \prime} T \in M$ which is a subset of $j(T)$ with cardinality less than $j(\kappa)$ in $M$. By elementarity and the assumption for $T$ in $V$, one can have a Boolean-valued model of $j^{\prime \prime} T$ in $M$ which can be easily translated to that of $T$. We go to a forcing extension $M^{\mathbb{P}}$ for $\mathbb{P}=\operatorname{Coll}(\omega, T)$ where $T$ can be coded by a real and argue that $T$ has a Boolean-valued model also in $M^{\mathbb{P}}$. By the soundness of $\Omega$-logic with real parameters, the statement " $T$ has a full second-order model" is $\Omega$-consistent with a parameter coding $T$ as a real. Then we argue that this is also true in $V^{\mathbb{P}}$ using the agreement of $\Omega$-logic in $V^{\mathbb{P}}$ and $M^{\mathbb{P}}$. Finally, we use the strong $\Omega$-conjecture to have $\Omega$-satisfiability of the statement " $T$ has a full second-order model" in $V^{\mathbb{P}}$ that implies that $T$ has a Boolean-valued model in $V$.

To ensure the agreement of $\Omega$-logic in $V^{\mathbb{P}}$ and $M^{\mathbb{P}}$, we will use the following lemma. Note that $V_{\eta}=M_{\eta}$ since $j \uparrow V_{\eta} \in M$.

Lemma 9.11 (Woodin) In the above situation, for any partial order $\mathbb{P}$ in $V_{\eta}$, the set of all universally Baire sets in $V^{\mathbb{P}}$ is exactly the same as that in $M^{\mathbb{P}}$. Hence the $\Omega$-logic with real parameters in $V^{\mathbb{P}}$ coincides with that in $M^{\mathbb{P}}$.

Proof of Lemma 9.11 See [4, Lemma 3.4.15].

We now proceed with the details of the arguments. Since $j \uparrow V_{\eta}$ is in $M$ and $T \in V_{\eta}$, $j^{\prime \prime} T$ is also in $M$ and it has cardinality less than $\eta<j(\kappa)$ in $M$. By the assumption, any subset of $T$ with cardinality less than $\kappa$ has a Boolean-valued model in $V$. Since $\left|j^{\prime \prime} T\right|<j(\kappa)$ in $M$, by elementarity of $j, j^{\prime \prime} T$ has a Boolean-valued model in $M$ as well.

Since $j \uparrow T$ is in $M$, one can easily transform a Boolean-valued model of $j^{\prime \prime} T$ in $M$ to that of $T$ in $M$ by sending the interpretation of symbols in $j^{\prime \prime} T$ to that of the corresponding symbols in $T$ via $j$. Hence $T$ also has a Boolean-valued model in $M$.

Let $\mathbb{P}=\operatorname{Coll}(\omega, T)$.

\section{Claim $2 T$ has a Boolean-valued model also in $M^{\mathbb{P}}$.}

Proof of Claim 2 Let $\delta>\eta$ be a Woodin cardinal in $M$ such that for any $\Sigma_{2}$-formula $\varphi$ and a real $x$ in $M$, if the sentence $\varphi(x)$ is true in some generic extension of $M$, then there exist $\alpha<\delta$ and $\mathbb{Q} \in M_{\delta}$ such that $M_{\alpha}^{\mathbb{Q}} \vDash \varphi(x)$.

Let $g$ be $\mathbb{P}$-generic over $M$. We will show that $T$ has a Boolean-valued model in $M[g]$. Since $\mathbb{P}$ is in $M_{\delta}$, there is a $\mathbb{P}_{<\delta}$-generic filter $G$ over $M$ such that $g \in G$.

Then $T$ is countable in $M[G]$ and there is an elementary embedding $k: M \rightarrow N \subseteq$ $M[G]$ such that $N^{<\delta} \cap M[G]<N$ with $k(\delta)=\delta$. By elementarity of $k$, in $N$, there is a Boolean-valued model of $k(T)$ and hence also a Boolean-valued model of $k^{\prime \prime} T$. Since $T$ is countable in $M[G]$, it is in $N$ and by using $k$, one can easily transform a Boolean-valued of $k^{\prime \prime} T$ to that of $T$ in $N$.

Note that the statement "there is a full second-order model of $T$ " is a $\Sigma_{2}$-statement with parameter $x$ in $N$. Then by the choice of $\delta$ and the elementarity of $k$, there exist $\alpha<k(\delta)=\delta$ and $\mathbb{Q} \in N_{\delta}=M[G]_{\delta}$ such that $N_{\alpha}^{\mathbb{Q}}$ satisfies that "there is a full second-order model of $T$." Since $N_{\delta}=M[G]_{\delta}$, it is also true in $M[G]$. Therefore, $T$ has a Boolean-valued model in $M[G]$ and hence in $M[g]$ for any $\mathbb{P}$-generic $g$ over $M$, as desired. 
We now have that $T$ has a Boolean-valued model in $M^{\mathbb{P}}$ where $T$ can be coded by a real. Then by the soundness of $\Omega$-logic with real parameters, the statement " $T$ has a full second-order model" (with a real parameter coding $T$ ) is $\Omega$-consistent in $M^{\mathbb{P}}$. By Lemma 9.11, the same statement is $\Omega$-consistent in $V^{\mathbb{P}}$. But since the strong $\Omega$-conjecture is true in $V$, the $\Omega$-conjecture with real parameters is true in $V^{\mathbb{P}}$. Hence the statement " $T$ has a full second-order model" is $\Omega$-satisfiable, that is, $T$ has a Boolean-valued model in $V^{\mathbb{P}}$ and hence in $V$, as desired.

We now discuss the Hanf numbers of Boolean-valued second-order logic and of full second-order logic. First we define the notion of cardinality of a Boolean-valued structure. Given a Boolean-valued structure $M=\left(A, \mathbb{B},\left\{R_{i}^{M}\right\}\right)$, the cardinality of $M$ is defined to be that of $A$.

For a given logic $L^{*}$, the Hanf number of $L^{*}$ is the least cardinal $\kappa$ such that if a sentence $\varphi$ in $L^{*}$ has a model of cardinality at least $\kappa$, then $\varphi$ has models with arbitrarily large cardinalities; that is, for any $\lambda, \varphi$ has a model with cardinality bigger than $\lambda$. For a sentence $\varphi$ in $L^{*}$, let

$$
\operatorname{sp}(\varphi)=\{\kappa \mid \varphi \text { has a model of cardinality } \kappa\} .
$$

We call $\operatorname{sp}(\varphi)$ the spectrum of $\varphi$. The Hanf number of $L^{*}$ is the least $\kappa$ such that for any sentence $\varphi$ in $L^{*}$ with bounded spectrum and $\lambda \in \operatorname{sp}(\varphi), \lambda<\kappa$.

We use $h^{2}$ and $h^{2 b}$ for the Hanf numbers of full second-order logic and of Boolean-valued second-order logic.

The following is due to Magidor.

Theorem 9.12 (Magidor) The Hanf number $h^{2}$ is between the first supercompact cardinal and the first extendible cardinal.

\section{Proof See [6].}

We show that $h^{2 b}$ is below the first supercompact cardinal and hence strictly smaller than $h^{2}$ under the same assumptions of Theorem 9.10.

Theorem 9.13 Assume a proper class of Woodin cardinals and that the strong $\Omega$-conjecture holds. Let $\kappa$ be a supercompact cardinal. Then $h^{2 b}<\kappa$.

Proof Since there are only countably many second-order sentences, it is enough to show that for each second-order sentence $\varphi$ with bounded spectrum for the Booleanvalued second-order logic, $\sup (\operatorname{sp}(\varphi))<\kappa$.

We will use Theorem 9.10. Let $\varphi$ be a second-order sentence with $\sup (\operatorname{sp}(\varphi)) \geq \kappa$. We will show that $\operatorname{sp}(\varphi)$ is unbounded. Given any cardinal $\lambda \geq \kappa$, we add $\lambda$-many constants $c_{\alpha}$ in the language of $\varphi$ and let $T$ be the set of sentences $\varphi$ and those stating $c_{\alpha} \neq c_{\beta}$ for all $\alpha<\beta<\lambda$. Since $\sup (\operatorname{sp}(\varphi)) \geq \kappa$, any subset of $T$ with cardinality less than $\kappa$ has a Boolean-valued model. Hence, by Theorem 9.10, the whole $T$ has a Boolean-valued model and that Boolean-valued model has cardinality at least $\lambda$, as desired.

Corollary 9.14 Assume a supercompact cardinal and a proper class of Woodin cardinals and that the strong $\Omega$-conjecture holds. Then $h^{2 b}<h^{2}$.

Note that $h^{2 b}$ is at least as large as $\kappa(\omega)$, if such exists, since well-ordering can be described in Boolean-valued second-order logic. 
Question 9.15 Can we say more about the least cardinal $\kappa$ such that $\kappa$ is $\mathrm{L}_{\kappa, \kappa}^{2 b}$-compact? How about the lower bound of Hanf number $h^{2 b}$ ?

Finally, we discuss Löwenheim numbers of Boolean-valued second-order logic and full second-order logic. Given a logic $L^{*}$, the Löwenheim number of $L^{*}$ is the least cardinal $\kappa$ such that for any sentence $\varphi$, if there is a model of $\varphi$, then there is one such with cardinality at most $\kappa$. Let us use $\ell^{2 b}$ and $\ell^{2}$ to denote Löwenheim numbers of Boolean-valued second-order logic and full second-order logic, respectively.

The following is a well-known characterization of Löwenheim number of full second-order logic.

Proposition 9.16 (Folklore) We have

$$
\ell^{2}=\sup \left\{\alpha \mid \alpha \text { is } \Delta_{2} \text {-definable in set theory }\right\} .
$$

In particular, $\ell^{2}$ is bigger than the least limit of Woodin cardinals if there is a proper class of Woodin cardinals.

Proof See, for example, [14].

We now show that $\ell^{2 b}$ could be smaller than $\ell^{2}$.

Theorem 9.17 Assume that the theory ZFC+ "There is a proper class of Woodin cardinals" is consistent. Then so is ZFC + "There is a proper class of Woodin cardinals and $\ell^{2 b}$ is less than the first Woodin cardinal." In particular, it is consistent that $\ell^{2 b}<\ell^{2}$. $^{2}$

Proof We use some facts from inner model theory. For the notions such as "minimal canonical inner model" and "Q2-structures," one can refer to [8] and [13].

We look at the minimal canonical inner model $M=\mathrm{L}[\vec{E}]$ with a proper class of Woodin cardinals and argue that $\ell^{2 b}$ is less than the first Woodin cardinal in $M$. Since one can prove that such an $M$ exists assuming ZFC + "There is a proper class of Woodin cardinals," this is enough to prove for the conclusion of the theorem. (For the proof of this fact, one can look at [8] and [13].)

We work in $M$. Let $\delta$ be the least Woodin cardinal in $M$, and let $\varphi$ be a secondorder sentence such that $\varphi$ has a Boolean-valued model. We will show that $\varphi$ has a Boolean-valued model with size less than $\delta$.

Let $\kappa$ be the least inaccessible cardinal above $\delta$ in $M$. Let $\varepsilon \subseteq M_{\delta}$ be a collection of nice extenders witnessing $\delta$ is Woodin in $M$. Let $X$ be a countable substructure of $M_{\kappa}$ such that $\varepsilon \in X$, and let $M_{X}$ be the transitive collapse of $X$. We will write $\bar{a}$ for the transitive collapse of $a$ in $X$ (e.g., $\bar{\delta}$ ). By Theorem 0.2 in Schindler and Steel [10], $M_{X}$ has an $\omega_{1}$-iteration strategy for nice iteration trees whose extenders come from $\overline{\mathcal{E}}$ which is led by $\mathcal{Q}$-structures, and hence it is universally Baire in the codes in $M$. Therefore, by Lemma 9.9, the $\Omega$-conjecture holds in $M$ and the statement $\psi \equiv$ " $\varphi$ has a full second-order model" is $\Omega$-consistent, that is, its negation is not $\Omega$-provable.

Let $\Sigma$ be the $\omega_{1}$-iteration strategy on $M_{X}$ we discussed in the last paragraph. Since $\Sigma$ is universally Baire in the codes and $\psi$ is $\Omega$-consistent, there exists a countable transitive model $N$ of ZFC such that $M_{X}$ is countable in $N$ and $N$ is $\Sigma$-closed. In particular, there exists a $\gamma$ in $N$ such that $V_{\gamma}^{N}$ has a Boolean-valued model of $\varphi$ whose first-order universe is in $V_{\gamma}$. 
We now use the extender algebra $\mathcal{W}_{\bar{\delta}, \bar{\delta}} \subseteq V_{\bar{\delta}}^{M_{X}}$ over $M_{X}$ (e.g., see Section 9 in [5]) to make $V_{\gamma}^{N}$ generic over an iterate $M_{\infty}$ of $M_{X}$ in $N$. Then $M_{\infty}$ has a Boolean-valued model of $\varphi$ whose first-order universe is in $V_{\delta^{\prime}}^{M_{\infty}}$, where $\delta^{\prime}$ is the image of $\bar{\delta}$ via the iteration map $\pi: M_{X} \rightarrow M_{\infty}$. By elementarity of $\pi$ in $M_{X}$, there exists a Boolean-valued model of $\varphi$ whose first-order universe is in $V_{\bar{\delta}}^{M_{X}}$. Finally, since $M_{X}$ was the transitive collapse of $X$, in $M_{\kappa}$, there exists a Boolean-valued model of $\varphi$ whose first-order universe is in $M_{\delta}$. Hence $\varphi$ has a Boolean-valued model with size less than the least Woodin $\delta$, as desired.

Question 9.18 Could $\ell^{2 b}$ be below the least measurable cardinal?

\section{Notes}

1. The definition of $\Omega$-validity here is different from Woodin's original definition in [17]. However, they are essentially the same in the following sense: There are recursive translations $\varphi \mapsto \psi_{\varphi}$ and $\psi \mapsto \varphi_{\psi}$ from $\Pi_{2}$-sentences to sentences and sentences to $\Pi_{2}$-sentences, respectively, such that a $\Pi_{2}$-sentence $\varphi$ is $\Omega$-valid in our sense if and only if $\psi_{\varphi}$ is $\Omega$-valid in Woodin's sense, and a sentence $\psi$ is $\Omega$-valid in Woodin's sense if and only if $\varphi_{\psi}$ is $\Omega$-valid in our sense. We use our formulation because it is simpler to work with when considering $\Pi_{2}$-statements.

2. There is a notion called " $A$-closure," weaker than strong $A$-closure, but there is no essential difference between them in our context. For the details, one can refer to [1].

3. The definition of $\Omega$-provability here is different from Woodin's original definition of $\Omega$-provability in [17]. However, they are essentially the same in the following sense: There are recursive translations $\varphi \mapsto \psi_{\varphi}$ and $\psi \mapsto \varphi_{\psi}$ from $\Pi_{2}$-sentences to sentences and sentences to $\Pi_{2}$-sentences, respectively, such that a $\Pi_{2}$-sentence $\varphi$ is $\Omega$-provable in our sense if and only if $\psi_{\varphi}$ is $\Omega$-provable in Woodin's sense, and a sentence $\psi$ is $\Omega$-provable in Woodin's sense if and only if $\varphi_{\psi}$ is $\Omega$-provable in our sense. We use our formulation because it is simpler to work with.

4. Since $M$ satisfies the comprehension axiom for each second-order formula and $A$ is infinite in $M$, such a structure exists in $\mathscr{G}$ uniquely up to isomorphism.

5. Such a $\rho$ exists because $M$ is an $\omega$-model.

6. $\quad N$ is a countable transitive model of a fragment of ZFC and one can define the strong $A$-closure for such a model in the same way as Definition 6.2.

7. The authors would like to thank John Steel for pointing out the corresponding result in $\Omega$-logic.

\section{References}

[1] Bagaria, J., N. Castells, and P. Larson, "An $\Omega$-logic primer," pp. 1-28 in Set Theory, Trends in Mathematics, Birkhäuser, Basel, 2006. MR 2267144. DOI 10.1007/3-7643-7692-9_1. 168, 177, 188 
[2] Bell, J. L., Boolean-valued Models and Independence Proofs in Set Theory, 2nd edition, vol. 12 of Oxford Logic Guides, Oxford University Press, New York, 1985. MR 0816941. 168

[3] Henkin, L., "Completeness in the theory of types," Journal of Symbolic Logic, vol. 15 (1950), pp. 81-91. Zbl 0039.00801. MR 0036188. 167

[4] Larson, P. B., The Stationary Tower: Notes on a Course by W. Hugh Woodin, vol. 32 of University Lecture Series, American Mathematical Society, Providence, 2004. MR 2069032. 174, 184, 185

[5] Larson, P. B., "Three days of $\Omega$-logic," Annals of the Japan Association for Philosophy of Science, vol. 19 (2011), pp. 57-86. MR 2857737. 168, 174, 176, 177, 183, 188

[6] Magidor, M., "On the role of supercompact and extendible cardinals in logic," Israel Journal of Mathematics, vol. 10 (1971), pp. 147-57. Zbl 0263.02034. MR 0295904. $169,181,186$

[7] Martin, D. A., and J. R. Steel, "Iteration trees," Journal of the American Mathematical Society, vol. 7 (1994), pp. 1-73. MR 1224594. DOI 10.2307/2152720. 182

[8] Mitchell, W. J., and J. R. Steel, Fine Structure and Iteration Trees, vol. 3 of Lecture Notes in Logic, Springer, Berlin, 1994. MR 1300637. DOI 10.1007/978-3-662-21903-4. 182, 183,187

[9] Rasiowa, H., and R. Sikorski, The Mathematics of Metamathematics, 3rd edition, vol. 41 of Monografie Matematyczne, PWN_Polish Scientific, Warsaw, 1970. MR 0344067. 168

[10] Schindler, R., and J. R. Steel, “The self-iterability of L[E],” Journal of Symbolic Logic, vol. 74 (2009), pp. 751-79. MR 2548477. DOI 10.2178/js1/1245158084. 187

[11] Schlutzenberg, F. S., Measures in mice, Ph.D. dissertation, University of California, Berkeley, Berkeley, Calif., 2007. MR 2711515. 183

[12] Steel, J. R., "Local $K^{c}$ constructions," Journal of Symbolic Logic, vol. 72 (2007), pp. 721-37. MR 2354897. DOI 10.2178/js1/1191333838. 183

[13] Steel, J., "An outline of inner model theory," pp. 1595-1684 in Handbook of Set Theory, Vol. 3, Springer, Dordrecht, 2010. Zbl 1198.03070. MR 2768698. DOI 10.1007/978-1-4020-5764-9_20. 176, 182, 183, 187

[14] Väänänen, J., "Abstract logic and set theory, I: Definability,” pp. 391-421 in Logic Colloquium '78 (Mons, 1978), vol. 97 of Studies in Logic and the Foundations of Mathematics, North-Holland, Amsterdam, 1979. MR 0567682. 169, 187

[15] Väänänen, J., "Second-order logic and foundations of mathematics," Bulletin of Symbolic Logic, vol. 7 (2001), pp. 504-20. MR 1867954. DOI 10.2307/2687796. 167, 168, 173

[16] Väänänen, J., "Second-order logic or set theory?," Bulletin of Symbolic Logic, vol. 18 (2012), pp. 91-121. MR 2798269. 168

[17] Woodin, W. H., The Axiom of Determinacy, Forcing Axioms, and the Nonstationary Ideal, vol. 1 of de Gruyter Series in Logic and its Applications, Walter de Gruyter, Berlin, 1999. MR 1713438. DOI 10.1515/9783110804737. 168, 188

[18] Woodin, W. H., "Suitable extender models I," Journal of Mathematical Logic, vol. 10 (2010), pp. 101-339. Zbl 1247.03110. MR 2802084. DOI 10.1142/S021906131000095X. 184

\section{Acknowledgments}

The authors would like to thank the European Science Foundation for grant 4633 within the INFTY program. The research of the first author is supported by a postdoctoral fellowship for research abroad funded by the Japan Society for the Promotion of Science; the research of the second author is partially supported by Academy of Finland grant 251557 and by the European Science Foundation EUROCORES LogICCC programme LINT project. The authors are grateful to W. Hugh Woodin for a number of discussions 
related to $\Omega$-logic. They would also like to thank the anonymous referees for many comments and suggestions for the paper.

Ikegami

Graduate School of System Informatics

Kobe University

Rokko-dai 1-1

Nada, Kobe 657-8501

Japan

ikegami@people.kobe-u.ac.jp

\section{Väänänen}

Department of Mathematics and Statistics

University of Helsinki

P.O. Box 68 (Gustaf Hällströmin katu 2b)

FI-00014 University of Helsinki

Finland

and

Institute of Logic, Language and Computation

University of Amsterdam

Science Park 107

1098 XG Amsterdam

The Netherlands

jouko.vaananen@helsinki.fi 